UCRL-ID-127383

\title{
Design of Software and Hardware Components for a Six-Degrees of Freedom Optical Position Sensor
}

\author{
F. N. Garcia
}

June 1, 1997

This is an informal report intended primarily for internal or limited external diatribution. The opinions and conclusions stated are thoce of the author and may or may not be those of the Laboratory.

Work performed under the auopices of the U.S. Department of Energy by the Lawrence Livermore National Laboratory under Contract W-7405-Eng-48. 


\section{DISCLAIMER}

This document was prepared as an account of work sponsored by an agency of the United States Government. Neither the United States Government nor the University of California nor any of their employees, makes any warranty, express or implied, or assumes any legal liability or responsibility for the accuracy, completeness, or usefulness of any information, apparatus, product, or process disclosed, or represents that its use would not infringe privately owned rights. Reference herein to any specific commercial product, process, or service by trade name, trademark, manufacturer, or otherwise, does not necessarily constitute or imply its endorsement, recommendation, or favoring by the United States Government or the University of California. The views and opinions of authors expressed herein do not necessarily state or reflect those of the United States Government or the University of California, and shall not be used for advertising or product endorsement purposes.

This report has been reproduced directly from the best available copy.

Available to DOE and DOE contractors from the Office of Scientific and Technical Information

P.O. Box 62, Oak Ridge, TN 37831

Prices available from (615) 576-8401, FTS 626-8401

Available to the public from the

National Technical Information Service

U.S. Department of Commerce

5285 Port Royal Rd.,

Springfield, VA 22161 
Design of Software and Hardware Components For A Six-Degrees of Freedom Optical Position Sensor

by

Fermin N. Garcia

Submitted to the Department of Mechanical Engineering in Partial Fulfillment of the Requirements for the Degree of

Bachelor of Science

at the

MASSACHUSETTS INSTITUTE OF TECHNOLOGY

June 1997

(c) 1997 Fermin N. Garcia. All Rights Reserved.

Author

Department of Mechanical Engineering April 28, 1997

Certified by

Seth Lloyd

Assistant Professor of Mechanical Engineering Thesis Supervisor

Accepted by

Peter Griffith

Professor of Mechanical Engineering Chairman, Department Committee on Undergraduate Students 


\title{
Design of Software and Hardware Components For A Six-Degrees of Freedom Optical Sensor
}

\author{
by \\ Fermin N. Garcia \\ Submitted to the Department of Mechanical Engineering on April \\ 28, 1997 in partial fulfillment of the requirements for the Degree of \\ Bachelor of Science in Mechanical Engineering
}

\begin{abstract}
This report summarizes the evaluation of a fully compatible and operational data acquisition system for a six-degrees of freedom optical sensor (SixDOF). The SixDOF, developed at Lawrence Livermore National Laboratory by Charles Vann [1], is capable of tracking an object's position in all its six degrees of freedom without any datum specification by means of two reflective surfaces mounted on the object. To make the SixDOF operational and thus validate its underlying physics, a signal processing system has been designed so that information from the sensor is transferred accurately and efficiently to a computer. In addition, a six-degrees of freedom positoning stage has been built in efforts to calibrate the sensor in real time. A crucial design constraint is the necessity to build the complete data acquisiton system so that it be small and most importantly portable.

The prototype of the SixDOF system proved to be capable of crudely detecting changes in the position of an object in all six spatial degrees of freedom. An accuracy of around 0.5 $\mathrm{mm}$ is estimated presently eventhough the position of the two reflectors on the object is seen to significantly influence the accuracy of the sensor. The resolution of the sensor is not quite understood yet because of uncertainties in the actual spot size of the laser, however, field of the view has been seen to increase as the resolution decreases. The decoupling (calibration) of the sensor data proved to be rather successful although some coupling still exists. This coupling, however, is almost certain to come from the crudeness in the alignment of the optics within the sensor.

Thesis Supervisor: Seth Lloyd Title: Associate Professor, Mechanical Engineering
\end{abstract}





\section{Table of Contents}

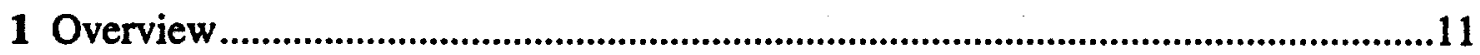

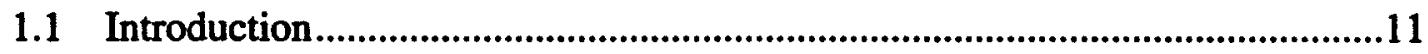

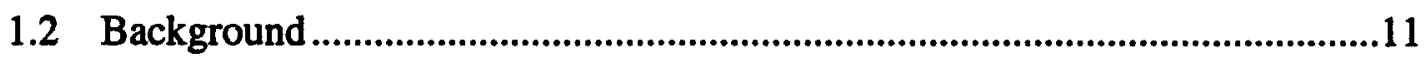

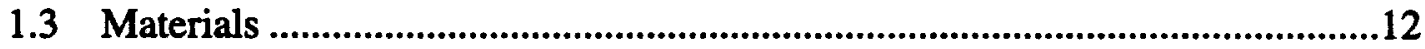

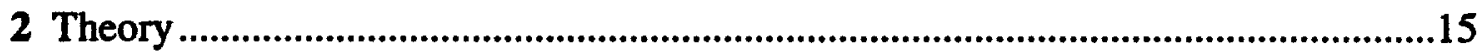

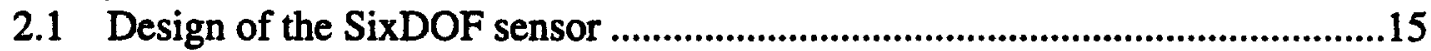

2.2 Design of the Graphical User Interface .....................................................19

2.3 Software Design for Data Acquisition .............................................................22

2.4 Design of the SixDOF Sensor Signal Processing System ...................................24

2.5 Design of the Six Degree of Freedom Positioner .................................................26

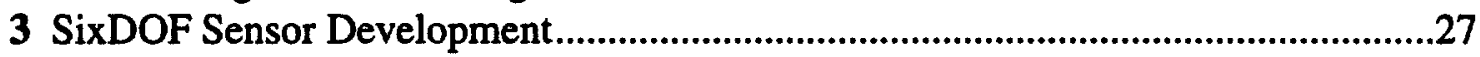

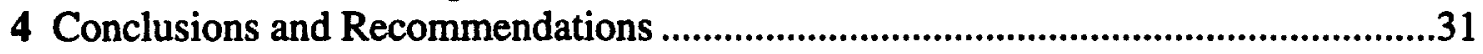

Appendix A Determination of the Calibration Matrix .......................................................33

Appendix B Wiring Schematics for Signal Processing Box ..................................................35

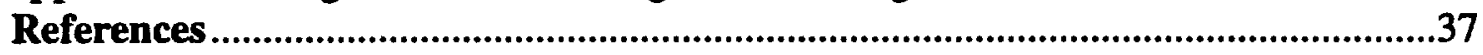




\section{List of Figures}

Figure 1.1: A schematic of the SixDOF sensor head........................................................13

Figure 2.1: Pin hole model for +lens showing sensitivity for $\mathrm{x}$ displacement..................15

Figure 2.2: Pin hole model for +lens showing slight sensitivity for tilt (Ry)..................16

Figure 2.3: Effect of $\mathrm{z}$ translation on position of light spot on PSD2 ..............................16

Figure 2.4: PSD1 is sensitive to $z$ translation. Rotation about $z$ axis has the same effect on the transversal axis of the PSD1. .....................................................................................17

Figure 2.5: Schematic showing sensitivity of PSD1 to $x$ translation; $y$ translation has the same effect on the transversal axis of PSD1.

Figure 2.6: Schematic shows sensitivity of tilt (Ry) on PSD1. The effect of tip (Rx) is identical but in the transversal direction of PSD1.

Figure 2.7: PSD3 is sensitive to $z$ translation. Since dot lies in the sensor's optical axis, rotation about the $\mathrm{z}$ axis cannot be detected. 18

Figure 2.8: Schematic shows the Graphical User Interface for SixDOF........................20

Figure 2.9: Schematic showing the VTX controls running the AD system.....................20

Figure 2.10: Display screen for SixDOF position data.......................................................21

Figure 2.11: Photograph of signal processing circuitry housing. ......................................25

Figure 3.1: Schematic of initial signal processing circuitry. ............................................27

Figure B.1: Wiring schematic for PSDs and Boards ........................................................35

Figure B.2: Pin schematic for Signal Processing Boards ..................................................36

Figure B.3: Schematic of 37 pin connector ......................................................................36 


\section{List of Tables}

Table 1.1: Comparison of the SixDOF and its closest competitor, Servo Robot's BIP35. 12 


\section{Chapter 1}

\section{Overview}

\subsection{Introduction}

A sensor is being developed at Lawrence Livermore National Laboratory that will be capable of measuring position in all six degrees of freedom. When placed on a robot or any type of automated machinery this Six Degrees of Freedom (SixDOF) sensor can be used to track the position of any object without the need for datum specification. The SixDOF can be of great use in the fields of automation and manufacturing. Currently, many automated assembly lines rely on datum specification of different axis, requiring robots to be given information about specific orientations and/or location of parts. This results in very long set-up times that can reduce throughput and limit the production capabilities of a plant. Therefore, a device, such as the SixDOF, that can incorporate the measuring capabilities to detect simultaneously all of the six degrees of motion would be a great asset to a manufacturer. For instance, the SixDOF can save a manufacturing plant the expense of designing jigs and fixtures for the specific positioning of parts in an assembly line. This allows for a more flexible manufacturing operation that is much more efficient and profitable.

The first significant step in the development process is to decouple all of the six degrees of freedom of information before any tests for practicability are done. In preparation for this process it has been necessary to design the electronic hardware and software to be used to make the SixDOF system operational. The first phase of development of the SixDOF began with a crude prototype of the sensor and culminated in a more refined prototype that was integrated with highly specialized signal processing equipment.

\subsection{Background}

As mentioned earlier, much time and money can be ill wasted due to inflexibility of manufacturing operations. For example, in the welding process of automobile doors, robots are required to be "walked" through the new sequence of operations if a design change is made or if a different part is to be fabricated. Besides being time consuming, this process is often inaccurate; this often leads to the use of expensive sensors and positioning devices. Some of this expensive technology includes non-contact laser sensors, one dimensional range detectors, and Miniature Laser Range Cameras (such as Servo Robot's Inc., BIP 35) that detect all 3 translations of a stationary object. The SixDOF is an improvement over all these competitive products since most importantly it eliminates the need for machine "training" and thus prevents costly production delays. For the case of automobile welding processes the SixDOF would be ideal since welding requires 3 degrees of freedom information to locate the weld site and three additional degrees of freedom to properly orient the tool with respect to the part. Some more key features of the SixDOF that are not currently available on the market are listed below (See Table 1.1, "Comparison of the SixDOF and its closest competitor, Servo Robot's BIP35.," pg. 12). 
Table 1.1: Comparison of the SixDOF and its closest competitor, Servo Robot's BIP35.

\begin{tabular}{l|l|l}
\hline $\begin{array}{l}\text { Characterist } \\
\text { ics }\end{array}$ & \multicolumn{1}{|c|}{ SixDOF } & \multicolumn{1}{c}{ BIP35 } \\
\hline Selling Cost & $-\$ 5000$ & $\$ 30,000$ \\
\hline Volume & $76 \mathrm{~cm}^{3}$ & $181 \mathrm{~cm}^{3}$ \\
\hline Weight & $85 \mathrm{~g}$ & $400 \mathrm{~g}$ \\
\hline Speed & $\sim 16.6 \mathrm{kHz}$ & 60 images $/ \mathrm{s}$ \\
\hline $\begin{array}{l}\text { Versatility } \\
\text { Measures } \\
\text { tip, tilt, and } \\
\text { rotation) }\end{array}$ & Yes & No \\
\hline
\end{tabular}

Finally, the SixDOF would be the only sensor capable of determining all 6 degrees of freedom relative to another object. Current competitive sensors are only capable of detecting at most three degrees of motion.

\subsection{Materials}

The SixDOF sensor system is composed of five main assemblies. These consist of the laser illuminator, the beam splitting and directing optics, the position sensitive photo diodes (PSD's), the beam reflectors (in this case two mirrors), and the signal processing electronics. The sensor head contains all of these assemblies with the exception of the reflecting mirrors and the signal processing electronics (See Figure 1.1: on page 13). The laser source used in the sensor is a $5 \mathrm{~mW}$ diode laser at a frequency of $670 \mathrm{~nm}$. Two small mirrors are used to redirect the $1 \mathrm{~mm}$ diameter laser beam onto the optical axis (M1 and M2). While in the optical axis, the beam is then passed through two negative lenses (L1 and L2) which diverge the beam into a $2 \mathrm{~cm}$ diameter spot at a distance of $3.5 \mathrm{~cm}$ from the face of the sensor. A filter within the optical axis is used to block out ambient light and most other frequencies not produced by the laser. The two reflecting mirrors are positioned $3.5 \mathrm{~cm}+1$ $\mathrm{cm}$ from the face of the sensor. One mirror is shaped as a $3.5 \mathrm{~mm}$ diameter circle, while the other is a $2 \times 1 \mathrm{~mm}$ rectangular bar. Reflected laser light from the rectangular bar is redirected onto one PSD (PSD 1) using a third mirror (M3). The reflected light from the circle, however, passes through a beam splitter that splits the beam equally between two PSDs (PSD 2 and 3). A positive lens (L3) is used to refocus the circular shaped reflected light onto PSD 2.

The signal processing components that were integrated with the SixDOF are listed as follows: 


\section{Hardware}

- IBM compatible laptop computer (Toshiba model 410CS) with PCMCIA type II socket

- Keithley MetraByte DASCard 1001 analog/digital converter card of type II PCMCIA

- 6 degrees of freedom sensor (SixDOF) box and optics

- 6 degrees of motion positioner

- 2 Gender changer connectors

- $\pm 15 \mathrm{~V} /+5 \mathrm{~V}$ dual power supply

- 15 Pin to 37 Pin box housing to hold signal processing electronics

- 3 Hamamatsu Tetra-lateral type Position Sensitive Diodes (PSDs) (Model \# S5991)

- 3 Hamamatsu analog signal conditioners compatible with S5991 PSD's (Model \#C4758)

\section{Software}

- Microsoft Visual Basic software package

- Keithley MetraByte Visual Test Extensions software

- SystemSoft CardWorks Card and Socket Services

- Microsoft Excel 5.0 software package

Figure 1.1: A schematic of the SixDOF sensor head.

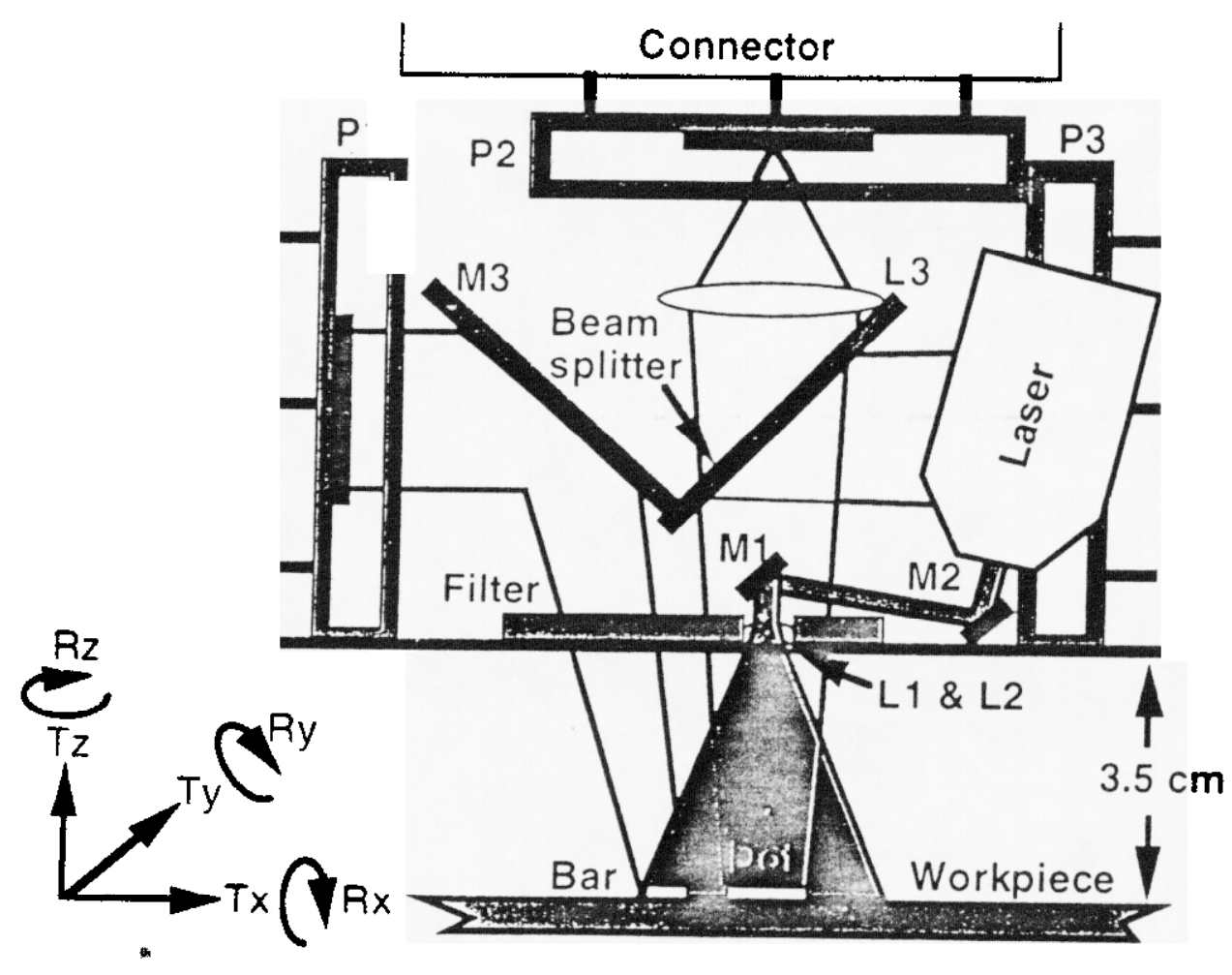




\section{Chapter 2}

\section{Theory}

\subsection{Design of the SixDOF sensor}

The SixDOF has three Position Sensitive Diodes (PSDs) that are each responsible for measuring position in two degrees of freedom. This is not to say that they are only sensitive to these two degrees of freedom and thus that is all they measure. Instead it is more accurate to say that they are affected by more than their two specific degrees of freedom, and at the end when all of them are coupled together each PSD gives information about two degrees of freedom. It is best to begin our discussion of the theory for the SixDOF by describing specifically the relation between the laser light, the reflectors, and the PSDs. Basically the sensor is setup so that collimated light emanating from the laser source is diverged with the negative lens and then bounced back from the reflectors (Mirrors 1 and 2) into the three PSDs. With the correct geometric alignment of the optics in this setup the PSDs are able to detect the motion of the reflections induced by these two reflectors as they are moved relative to the sensor head. This motion on the PSDs induce proportional changes in their voltage output and this can then be measured to give a relative position. It is the output from these three PSDs which contain the information about position in all six degrees of freedom. In fact it is not until this information is decoupled that all the six degrees are known.

The SixDOF relies on the optical properties of a divergent laser beam to detect the six degrees of motion simultaneously. It is this property of the laser light that when combined with simple lens and mirror theory gives the sensor its powerful capabilities. For instance, the reflection of the circular reflector on PSD2 is brought to a focus using a positive lens. It is the inherent properties of a positive (convex) lens that allow PSD2 to be highly sensitive to tilt and tip $\left(R_{x}\right.$ and $\left.R_{y}\right)$ and translation in $x$ and $y$, while not being sensitive to $Z$ translation and rotation $\left(R_{z}\right)$. Figures 2.1-2.3 below describes the physics of PSD2's setup.

Figure 2.1: Pin hole model for +lens showing sensitivity for $\mathrm{x}$ displacement.

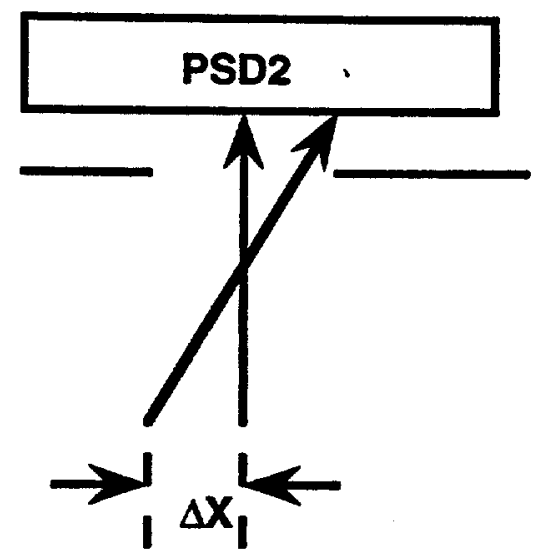


Figure 2.2: Pin hole model for +lens showing slight sensitivity for tilt $\left(R_{y}\right)$.

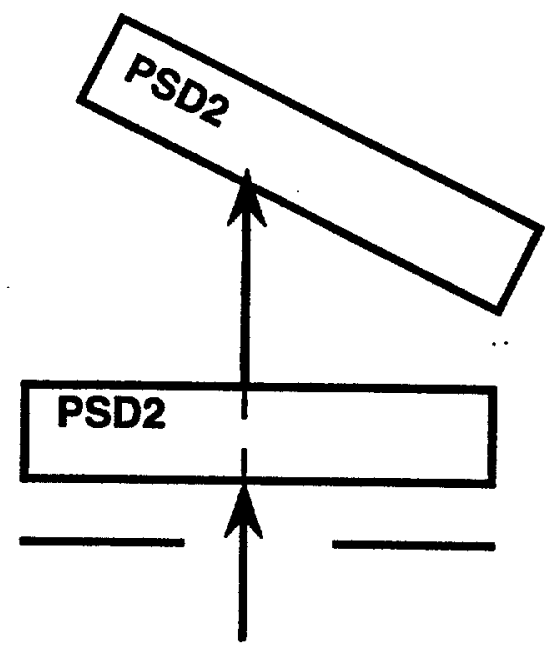

Figure 2.3: Effect of $\mathrm{z}$ translation on position of light spot on PSD2.

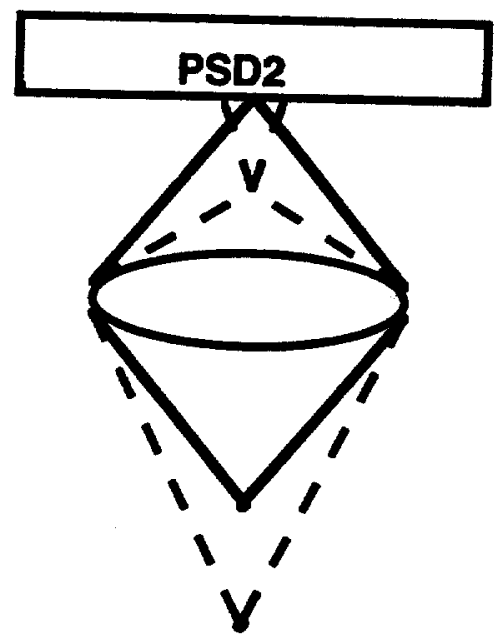

Note that PSD is not sensitive to $\mathrm{z}$ because motion in this axis only diverges the beam but does not displace the centroid of the light spot. It is this centroid that is detected. With the signal conditioning boards we are able to null out the PSD's sensitivity to light intensity and thus eliminate concerns of having the PSD output signal be spot size dependent. Also note that the reason PSD2 cannot detect $R_{\mathbf{z}}$ is because the reflector, by virtue of being symmetrical about the optical axis, induces an identical reflection when purely rotated about the optical axis. 
PSD1 and PSD3 also play critical roles in the sensor. The absence of a positive lens to focus the beam reflection causes PSD1 to be highly sensitive to $z$ translation. PSD3, unlike PSD1, is not sensitive to $R_{z}$ (as a matter of fact PSD1 is the only PSD that can detect $R_{z}$ ). This is because the reflection on PSD1 is the reflection from the bar shaped mirror and it is not entirely symmetrical about the optical axis (in fact the bar shaped mirror is slightly off the optical axis). Since PSD3 still sees a circular and axis-symmetrical reflection, a pure rotation about $\mathrm{z}$ cannot be detected. See Figures 2.4-2.6 below for schematic of the optics involved with PSD1.

Figure 2.4: $\mathrm{PSD} 1$ is sensitive to $\mathrm{z}$ translation. Rotation about $\mathrm{z}$ axis has the same effect on the transversal axis of the PSD1.

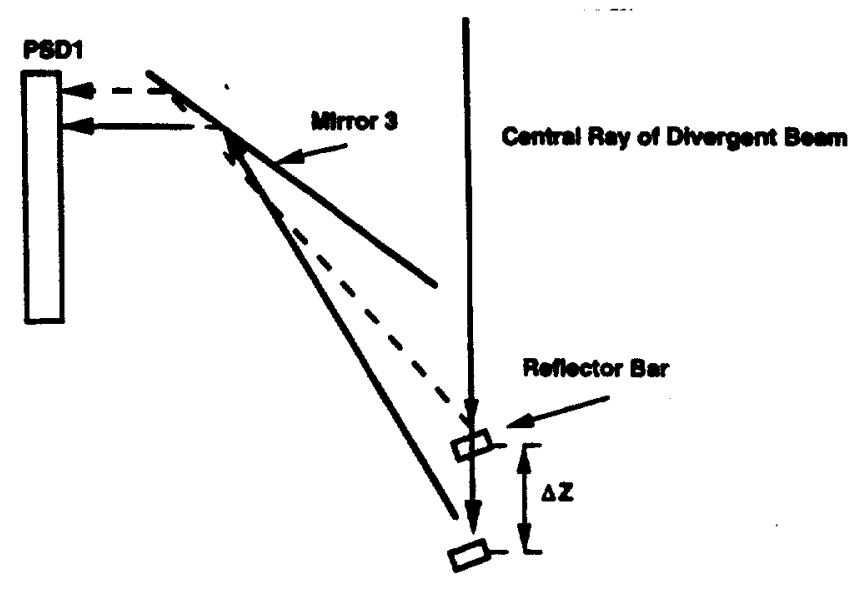

Figure 2.5: Schematic showing sensitivity of PSD1 to $x$ translation; $y$ translation has the same effect on the transversal axis of PSD1.

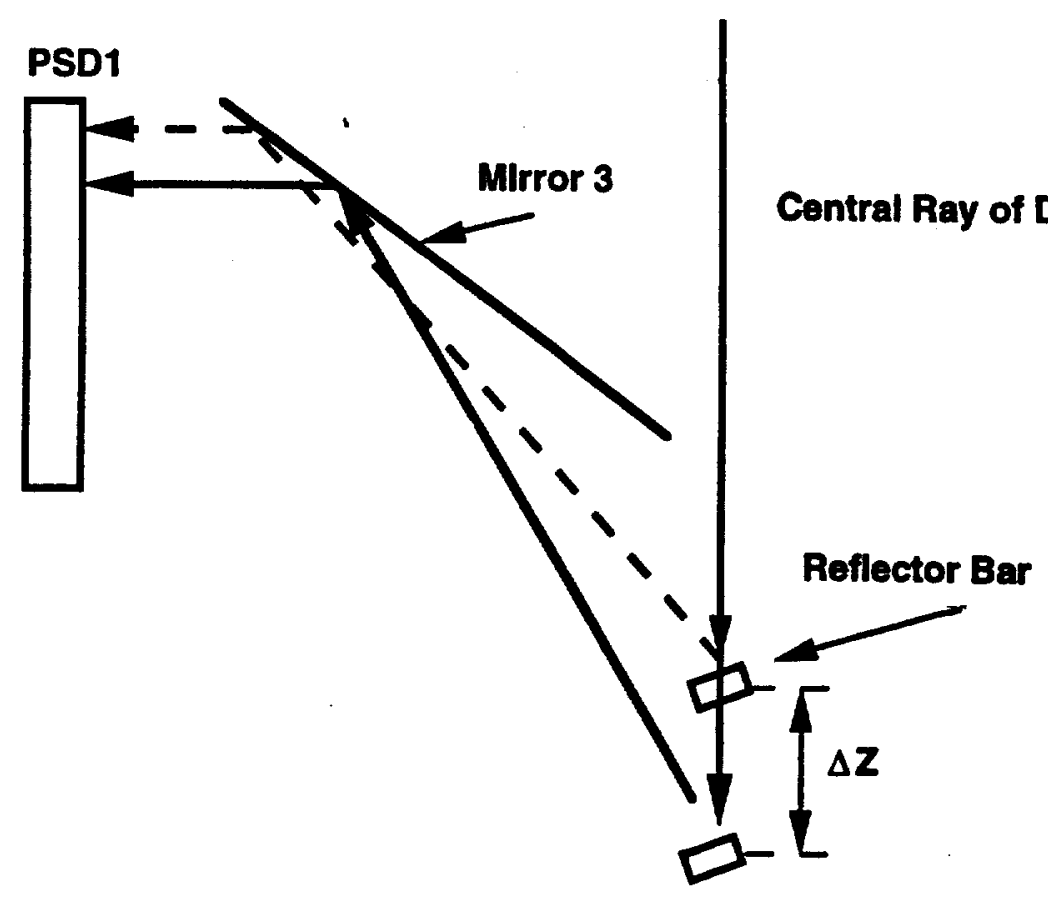


Figure 2.6: Schematic shows sensitivity of tilt $\left(R_{y}\right)$ on PSD1. The effect of tip $\left(R_{x}\right)$ is identical but in the transversal direction of PSD1.

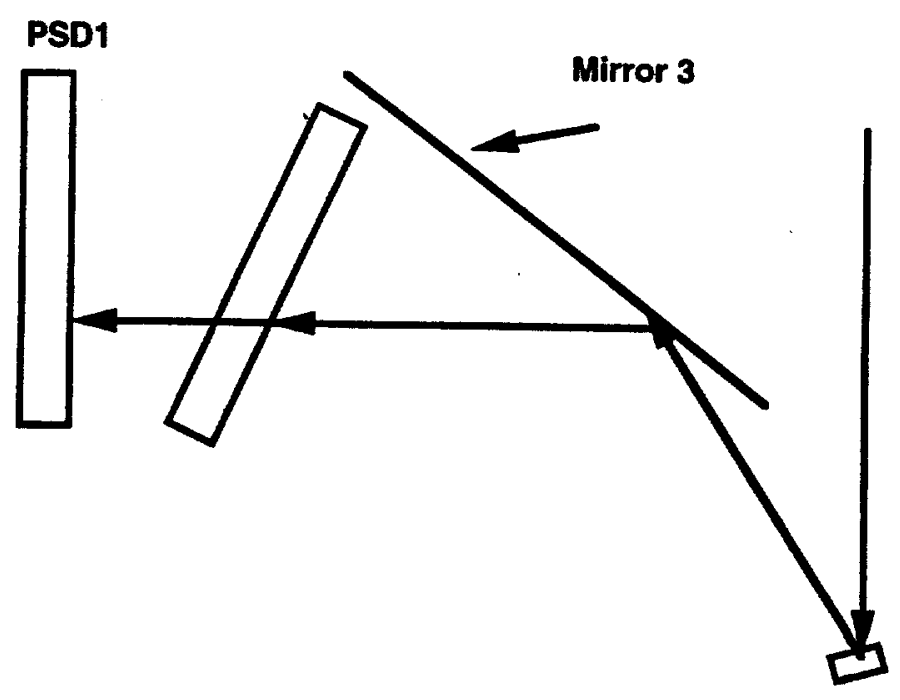

PSD3, like PSD1, also lacks a positive lens to refocus its image, but unlike PSD1 it is not sensitive to $\mathrm{z}$ translation. The reason for this being is that the dot reflector, to first order, is aligned with the beam splitter (the beam splitter is aligned with the optical axis of the sensor) that reflects the dots image on PSD3. As a result any $z$ displacement does not affect the way the beam splitter redirects the dot's image. See Figure 2.7 below for a drawing describing the optics involved with PSD3.

Figure 2.7: PSD3 is sensitive to $\mathrm{z}$ translation. Since dot lies in the sensor's optical axis, rotation about the $\mathrm{z}$ axis cannot be detected.

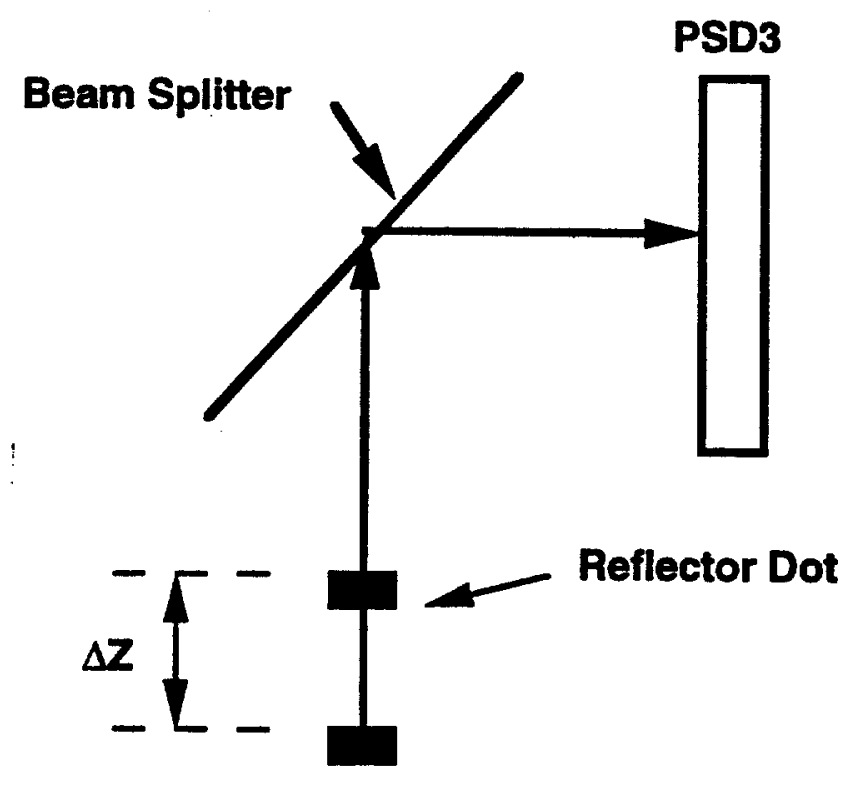


PSD3 is only affected by translation in the $\mathrm{x}$ and $\mathrm{y}$ direction and tilt and tip of the sensor head for the same reason PSD1 detects these (See Figure 2.5: on page 17, and Figure 2.6: on page 18). In summary, the combination of all three PSDs in the sensor can detect all the six degrees of freedom in space.

In efforts to decouple the information form all three PSDs it is necessary to determine a calibration matrix so that when readings are obtained, a straightforward linear algebra transformation can be done to yield the correct position data. The following mathematical procedure may be used to determine the calibration matrix of a deflection-sensing device having $\mathrm{j}$ sensors and $\mathrm{k}$ degrees of freedom. In the case of the SixDOF sensor system $\mathrm{j}=6$ and $k=6$. The reason for assuming $j=6$ is that there are three detectors (PSDs) inside of the sensor each giving two output readings. Each detector can then be treated as being equivalent to having two sensors that cross-talk with each other and with the other PSD output readings. The relation between sensor readings and deflections can be expressed as

$$
[C][S]^{2}=[D]^{\prime}
$$

Where the matrix $[\mathrm{C}]$ is a $\mathrm{kx} \mathrm{j}$ matrix of calibration terms relating sensor readings to actual deflections, [S] is a $\mathrm{n} \times \mathrm{j}$ matrix of sensor data where each row is a sample and each column is a set of $\mathrm{n}$ data points for a single sensor, and [D] $]^{\mathrm{t}}$ is a $\mathrm{k} \mathrm{x} \mathrm{n}$ matrix with each row representing one of $\mathrm{n}$ known deflections applied to the device and each column is a $\mathrm{k}$ element deflection vector (a vector that contains all the sensor readings for the nth sample). To determine $[\mathrm{C}]$ each row of the matrix is computed using a least squares fit as follows:

$$
\left([D]^{t}[D]\right)^{-1}[D]^{t}[S]=[C I]^{\prime}
$$

Since $\mathrm{j}=\mathrm{k}$ in our system the matrix $[\mathrm{CI}]$ can be inverted directly to get $[\mathrm{C}]$. From Equation 2 the transpose of $[\mathrm{CI}]^{t}$ can be computed to get $[\mathrm{CI}]$. [C] is then the calibration matrix for the sensor. Note that a matrix analysis is well justified because the small-deflections occur in a linear fashion. Using this procedure it is theoretically possible to eliminate any crosstalk between PSD measurements and thus arrive at six independent position components. This procedure is similar to the one used by Shimano [2] to decouple measurements from different components of deflection for the Scheinman Wrist. However, if the scatter and linearity of the data needs to be quantified the calibration matrix can be multiplied by the sensor readings and then the resulting positions can be compared with the original recorded positions.

\subsection{Design of the Graphical User Interface}

It is necessary in the design of our system, as well as in future applications, to use a Graphical User Interface (GUI) that can aid plant employees and machine operators in using the SixDOF sensor system. A relatively simple and effective GUI is implemented into the SixDOF system. Written in the Visual Basic programming language, it is responsible for both the control of data acquisition parameters and for the graphical display of the sensor readings (The GUI is also designed to check on the status of the electronics and of the lighting conditions inside the sensor). A schematic of the GUI is shown in Figure 2.8.

The GUI consists of two text boxes on the upper left hand side which allow setting of the A/D sampling parameters and three command buttons: a start button for initiating the data 
acquisition process, a stop button that halts the above process, and an exit button that allows the user to terminate and exit the SixDOF system (See Figure 2.8: on page 20).

Figure 2.8: Schematic shows the Graphical User Interface for SixDOF.

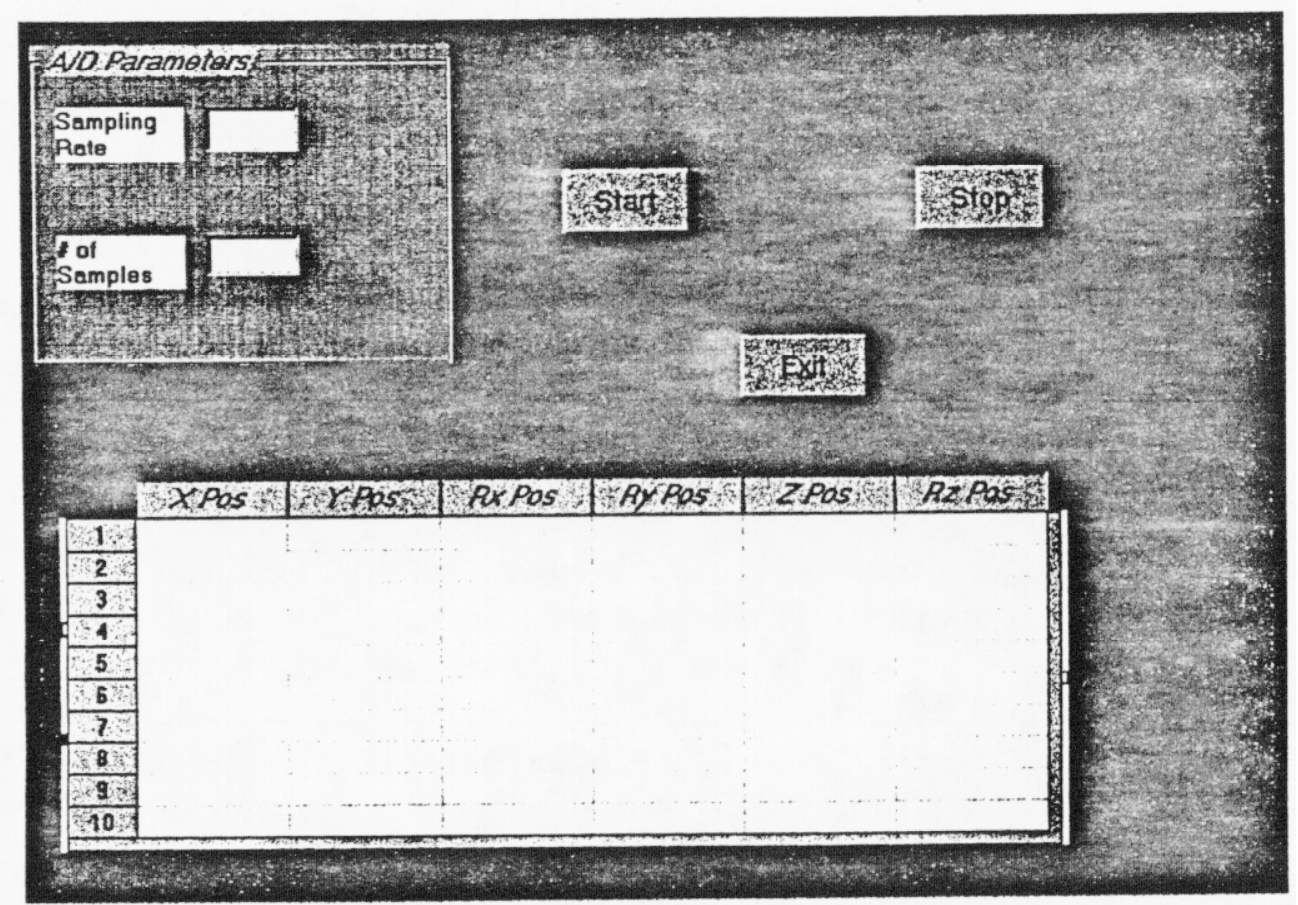

The GUI uses the Visual Test Extensions (VTX) software to perform the required data acquisition functions. VTX uses the information that the user inputs into the Visual Basic application programs such as the text boxes and the command buttons to run a data acquisition board installed in the computer. VTX can be thought of as the link between the Visual Basic command instructions and the A/D system. The VTX form designed for the system is shown in Figure 2.9.

Figure 2.9: Schematic showing the VTX controls running the AD system.

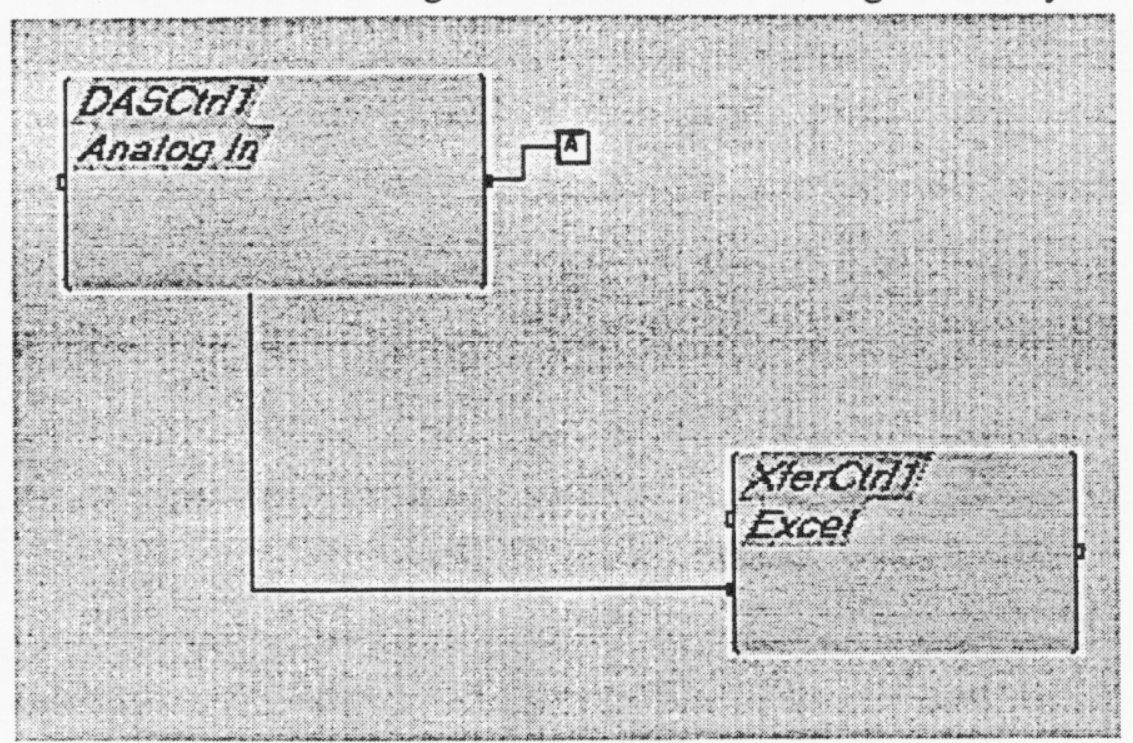


It shows a DASCtrll (DAS control) block representing the data acquisition board (A/D PCMCIA card), and a XferCtrll (Transfer control) block that represents the link between Microsoft Excel and VTX. Excel is used for filtering the signals coming from the sensor via the DASCtrll and for graphing the data of interest. Filtering is done averaging every five digital samples and using the average as the new value. In addition to filtering, the spreadsheet is used to read the data in a graphical format. See Figure 2.10 below for the schematic of the Excel spreadsheet that is linked to VTX.

The TextCtrll (Text control) is also part of the VTX environment, but unlike the DAS control and the Transfer control it is found on the GUI form. This is done this way because the Text control is primarily used to provide the user with a grid- like display of data sets. The Text control is implemented here solely for debugging purposes. It displays on the GUI form the raw data being read by the $A / D$ thus allowing the user to see problems before any erroneous filtering or graphing is done. The VTX format that is shown in Figure 2.9 is a simple block diagram schematic which sends sensor input data from the A/D card to the users screen. The lines represent actual data transfer connections. The line ending at the box marked " $A$ " connects the DAS control on the VTX form to the Text control on the GUI form. The Visual Basic programming language and the VTX environment work together to make a complete data acquisition application capable of meeting the needs of the hardware used in the SixDOF system.

Figure 2.10: Display screen for SixDOF position data.

Position in Six Degrees of Freedom

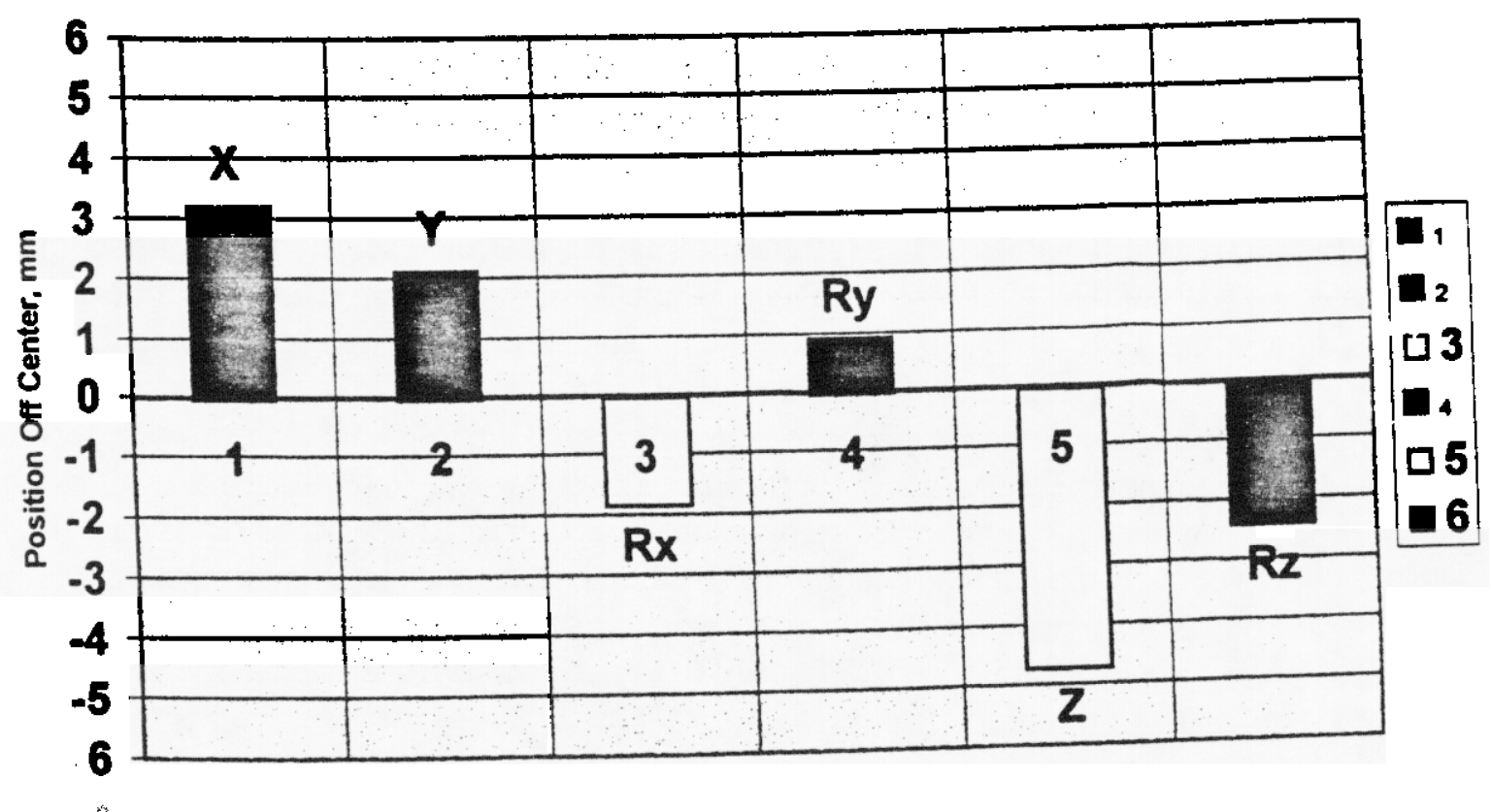




\title{
2.3 Software Design for Data Acquisition
}

\subsubsection{Visual Basic Instructions for Command Buttons}

The Visual Basic (VB) programming software can be described as fully event driven. This means that the language is structured such that code is executed only following the occurrence of an event. For example, the code written for the three command buttons in Figure 4 will be executed following the click of the left mouse button. In this specific case the event is the actual click of the mouse button and the code that will follow are the instructions to operate properly the data acquisition system. The following code is written in the Visual Basic programming language for the operation of the start button:

\author{
IPrivate Sub cmdStart_Click() \\ $2 Y=$ GUI_Form.TextCtrll.ClearInputs \\ 3Control_Form.DASCtrl1.Samples $=$ CLng(NumbText.Text) \\ 4Control_Form.DASCtrll.ConvRate $=$ CLng(SampText.Text) \\ 5Control_Form.DASCtrl1.ArmState $=1$ \\ 6GUI_Form.cmdStart.Enabled $=$ False \\ 7GUI_Form.cmdStop.Enabled $=$ True \\ 8GUI_Form.cmdStop.SetFocus
}

\section{End Sub}

It is clear that line 1 establishes the name of the command button in question and the event that executes the code, namely the click event. Line 2 clears all data from the Text control and assigns an event completion value to the variable $Y$. An event completion value is a feature of the Visual Basic environment that tells the user whether a certain task was actually completed. By default the system returns $Y=0$ if the specific task is not completed and $Y=-1$ if the task is complete. Thus, in line 2 the variable $Y$ is assigned the value 0 if the data in the Text control box is not cleared and -1 if it is. The user can use this feature with an if statement to check if any semantic errors exist. We did not implement this check since our application has simple algorithms and thus writing superfluous code would reduce its efficiency. The third and fourth lines allow the user to input desired values for the sampling rate and the number of samples to be acquired. It uses the VB library function CLng() to convert the data the user inputs in the two text boxes into real numbers. By design the text boxes only read variables as text, making the CLng() function essential for proper data conversion. In line 5, the DAS control (this item will be discussed in the following section) is instructed to commence the data acquisition. The value 1 represents that the status of the DAS control is set to "ignore connection". Since the DAS control lacks any connections to it, the command "ignore connection" has the effect of initiating its operation.

Lines 6 and 7 set the Text control and Transfer control so that they "wait for connections" before they commence with their respective tasks. A value of 0 for the ArmState property of any VTX control sets the status to "wait for connection". For instance, in the case of line 7 the Transfer control is told to wait for the connection from the DAS control before initi- 
ating operation. In other words, the Transfer control has to wait for the DAS control to finish its task before it sends data to the Excel spreadsheet.

Line 6 makes the start button inaccessible to the user so that upon clicking it once it can not be reclicked unless it is changed in code (See code for stop button). This prevents the data acquisition process from being interrupted during its operation. Experience shows that interruption of the normal operation of the VB data acquisition system can corrupt the data significantly. Line 7, however, makes the stop button accessible to the user. Line 8 thus highlights the stop button so that the user knows it is the next logical button to press. The exit button, however, can be pressed at any time during the data acquisition process. The stop button is responsible for stopping all data acquisition processes. The following Visual Basic code is written under the click event procedure of the stop button:

IPrivate Sub cmdStop_Click()

$2 X=$ Control_Form.DASCtrll.Halt

$3 Y=$ GUI_Form.TextCtrll.Halt

$4 Z=$ Control_Form.XferCtrl.Halt

5GUI_Form.cmdStop.Enabled $=$ False

6GUI_Form.cmdStart.Enabled $=$ True

7GUI_Form.cmdStart.SetFocus

\section{End Sub}

The "Halt" command in lines 2, 3 and 4 stop the operation of the DAS control, the Text control, and the Transfer control respectively. This is necessary in order to prevent process overruns. Process overruns usually happen when a sequence of controls running multiple times is disturbed by an instruction that is out of sequence. The returning of values for the variables $X, Y$, and $Z$ can provide the user with the status of any specific VTX control immediately prior to the Halt command. If the returning value is $\mathbf{0}$ the control status was inactive at the time of halt, but if the value is 1 the control was active. These return values were useful during the debugging phase when many of the halting instructions conflicted with one another. Moreover, the halt command automatically sets the value of the ArmState property of the VTX controls to 2. A value of 2 holds the VTX control in an inactive state until it is changed again only through code. Lines 5,6 , and 7 do the reverse of what lines 8,9 , and 10 do in the start button code. The combined effect of these three lines is to disenable the stop button while enabling and highlighting the start button.

The code for the exit button is self-explanatory. It only consists of one line of instruction. Namely the word "end". The exit button basically shuts down the data acquisition sytem entirely. Thus the code is as following:

1Private Sub cmdExit_Click()

2End

3End Sub 


\subsubsection{Visual Basic Instructions for VTX Controls}

As mentioned earlier the VTX controls consist of the DAS control, the Text control, and the Transfer control. Due to the nature of the VTX environment these three controls can handle autonomously the data acquisition functions with little code writing. By design these controls are generally able to pass and receive data without the need for writing code. For this reason, the DAS control and the Text control do not contain any Visual Basic code associated with data acquisition instructions. The transfer control, however, does have some Visual Basic code written for data acquisition functions. The following lines are the complete code for the Transfer control:

\section{IPrivate Sub XferCtrl1_ProcessDone(WarningCode As Integer)}

\section{Control_Form.DASCtrl1.ArmState $=1$ 3GUI_Form.TextCtrl1.ClearInputs 4End Sub}

As seen before, line 1 declares the control function used and the event that executes the code. The control function here is the Transfer control and the event is none other than the completion of its default tasks. Line 2 is responsible for turning on the DAS control again so that new data is read in from the sensors. One should note that an ArmState value of 1 only pulses the data acquisition board for a specified amount of time. Therefore it is necessary to trigger the DAS control every time data is transferred from the Transfer control to a spreadsheet. The VTX/VB system has an internal triggering mode but it relies on the sending of interrupts from the CPU to the application system. The result is a very slow and often comupting data acquisition process. Line 3 is responsible for clearing all the data from the text control after the completion of the data transferring process carried out by the Transfer control in order to place new data. The need to check for the completion of the clearing task is not implemented in the software because of the inefficiencies of this procedure. It should be noted that a synchronous mode with an internal clock source is used to have the data coming into the DASCtrll updated continuously. This mode along with the desired output channels (with their associated gain values) to be read, can be set using the properties table for the DAS control within Visual Basic application. Also of importance is the setting of the DAS control ArmState to a value of 2 (2 instructs any VTX control to "hold" until further change) and the setting of both the Text and Transfer control ArmState to "wait for control connection" (a value of 0 ). This allows the data acquisition process to start out smoothly and prevents it from process overruns. These settings are also done in the properties windows for these respective VTX controls. The reader should seek a VB and/or VTX reference manual if not familiar with this software

\subsection{Design of the SixDOF Sensor Signal Processing System}

In order to acquire position data from the three PSDs it is necessary to incorporate Hamamatsu's Analog Signal Processing Boards. The connection of the PSDs to the boards provides output voltages corresponding to the position data of a light spot traveling on the sensitive area of the detectors (See Appendix B for wiring diagrams). The position of the 
light spot is given with respect to a point of origin, namely the center of the PSD. In addition, the position data of the light spot can be obtained by simply replacing the unit of volts with millimeter. This signal processing circuitry also is designed so as to provide the true position data independent of light intensity. This proves very valuable in our SixDOF system because the optics inside the sensor provides various size light spots on the three PSDs. Thus in order to achieve consistent data from all three PSDs it is necessary to eliminate the PSDs dependence on light from the calibration and operation of the sensor.

Keeping in mind the required signal processing equipment and the design goal of keeping the SixDOF sensor as compact as possible, a junction box is designed so as to contain all the necessary signal processing circuitry and its associated peripherals. This keeps the relatively small optics and detectors inside the sensor while keeping the larger, more space consuming signal processing equipment remotely outside the sensor. See Figure 2.11 for schematic of the circuitry housing layout design.

Figure 2.11: Photograph of signal processing circuitry housing.

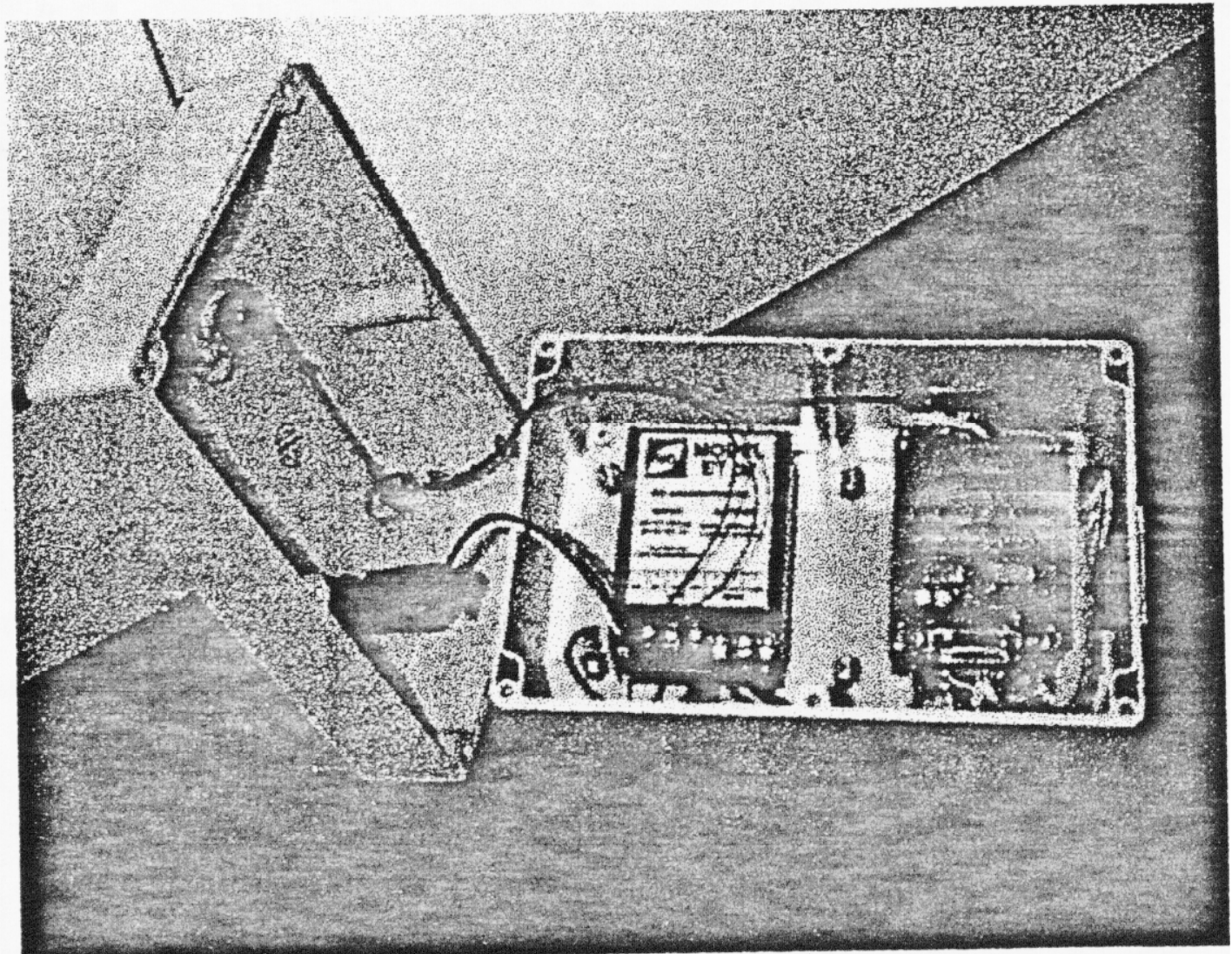




\subsection{Design of the Six Degree of Freedom Positioner}

The main purpose of the six degrees of freedom positioner is to serve as the calibrating device for the SixDOF sensor. The sensor is to be placed rigidly on this positioner and moved a known distance with respect to a stationary stage. The positioner itself is a fixture designed mostly out of spare aluminum parts from a variety of optic fixtures. The final design of this fixture consists of a mounting for the SixDOF sensor, a stage for placement of the reflectors, three precision micrometer dials, and three optic rotary dials. Using the six dials, position in all the six degrees of motion can be measured accurately.

A very important design objective for the positioner is the capability of the positioner to provide the sensor with pure rotations with respect to the $x, y$, and $z$ axis. This means that the sensor head rotates about the point in space where the three Cartesian axes intersect and the $\mathrm{z}$ axis coincides with the optical axis of the sensor. Meeting this condition prevents any $\mathrm{x}, \mathrm{y}$ or $\mathbf{z}$ translation component to be included in the rotation of the sensor head.

The procedure for aligning the sensor properly in the positioner consists of directing a laser beam at the surface of a reference spot attached near the center of any of the three optic rotary dials. The rotary dial is then rotated through a total arc of about $180^{\circ}$ while positioning the reference spot by trial and error until the laser beam remains on the spot throughout the entire rotation of the dial. This procedure is repeated for the other two rotary dials so as to find the true centers of rotation of the three dials. Positioning the center of the negative lens on the point at which the axis containing all three of these centers intersect yields the desired alignment. Now, one can guarantee that the positioner will give pure rotations to the sensor head. It should be noted that even though this procedure seems like a pretty crude way of aligning the sensor head, it actually is sufficiently accurate. The reason for this lies in the small rotations of the sensor during calibration and even actual operation. These rotations are on the order of milliradians and at these small movements the position of the laser beam on the reference spot does not move a significant amount such that it can be detected. Therefore, if the reference spot on the dials does not seem to move as they are rotated half a revolution, one can rest assured that when the dials are rotated a few milliradians the spot will move far less than what our detectors can resolve. 


\section{Chapter 3}

\section{SixDOF Sensor Development}

\subsection{Evolution of Electronics}

The purpose of this section is to provide a complete overview of the steps taken to bring the SixDOF sensor closer to being an operational position-sensing device. The very first steps of the development process consisted of defining and implementing the steps necessary to acquire and process the data coming from the sensor's three photodetectors. In this aim, the Graphical User Interface was designed along with the data acquisition system. In the way of the signal processing electronics, a very simple design was implemented consisting of a small junction box which connected each of the four PSD outputs to resistors and op-amps. The PSDs dependence on light intensity would be handled through software so as to make the electronics compatible with our SixDOF sensor. See Figure 3.1 below for a schematic of this initial junction box.

Figure 3.1: Schematic of initial signal processing circuitry.

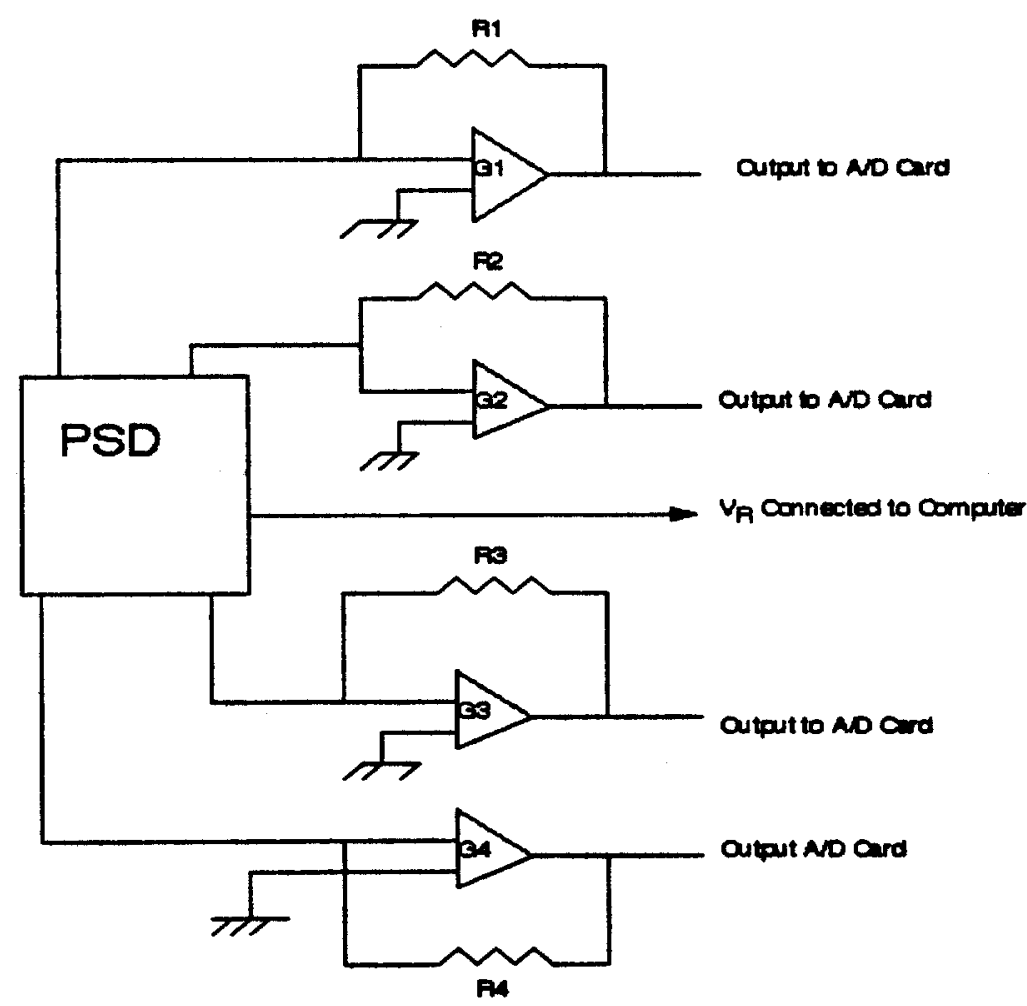

$A 1=F Q=P Q=P A=1 \mathrm{MP}$

$C 1=C R=C B=C 4=1000$ 
The use of the resistors is solely for providing a current to voltage transformation in the signal so a voltage can be measured with a reference to ground. The op-amps, however, are used to amplify the milliampere signal coming from the PSDs. This design seemed not to give convincing results as was seen by the random output signals observed. The data acquisition system was then tested with a millivolt calibrator in order to determine the cause for the nonsense readings coming from the PSDs. From these tests, it was determined that the data acquisition system was not the problem and that the signal processing electronics were not working as expected. The problem seemed to be that the electronics were somehow causing the 4 outputs of each PSD to track each other and not respond properly. Since the manufacturer of the PSDs designs a signal processing circuit specifically for the tetralateral type 2-D PSDs that are found in the sensor, it was decided to use these after failed efforts to build a simpler, less costly circuit.

Immediately after testing the new Hamamatsu circuit boards with our existent PSDs significant improvements were seen. One simple test that was setup consisted of connecting one PSD to one circuit board and then moving a light spot across the PSD while studying the two outputs from the board. The outputs from the board proved consistent with the motion of the light spot observed on the PSD. Even though we found a solution to this problem, other problems that were latent under the signal processing issues became apparent. One problem consisted of misalignments of the optics within the SixDOF sensor. In particular, it consisted of a misalignment between PSD1 and the mirror which redirects the beam upon it (M3). The way they were positioned relative to each prevented the reflected beam from reaching PSD1's sensitive area. As a result, PSD1 does not get a well defined light spot throughout its entire detection range. The mirror and the PSD were then adjusted accordingly so as to allow the beam to reach PSD1 and still be able to travel the entire sensitive area of the PSD. Also as the beam travel was studied more carefully it was noticed that the initial reflectors that were chosen (thin aluminum sheets cutout to the proper size and geometry as the current reflectors) were not of good quality and thus the image on the PSDs was not very focused. Large surrounding halos formed around the image being detected which tended to significantly degrade the accuracy of the data. When the aluminum sheets were replaced with the two reflector/mirrors of the exact shape and size, the image on the PSD improved significantly. Only a small halo was observed on PSD1 and it was attributed to the dirt on the upstream optics such as the negative lens, the filter, and M3. Another explanation for the formation of this halo is that M3 does not have a very flat surface and therefore the light wavefronts tend to get out of phase. This results in excess light scattering as the beam is redirected away from the mirror and unto PSD1. Our experiments show that it is not the unfocused large light spots that degrade the detectors accuracy, but instead it is the excess scatter of light. However, the present halo associated with the image on PSD1 is not of sufficient intensity in comparison to the image itself so as to affect the output of PSD1.

After determining the usefulness of the circuit boards for the PSDs, a new junction box was designed to house the three circuit boards and its power supply. Appendix B includes a detailed wiring diagram of the new junction box.

After fabrication of the new junction box the entire system was then ready for testing and debugging. Immediately it was noticed that excess noise was leaking into the system when the boards were powered but no inputs were given to the PSDs. Very frequent voltage spikes were seen in the data as well as magnitudes as high as +-5V. The specifications for 
the circuit board gave values for output noise 3 orders of magnitude lower than what was being seen. The noise, however, seemed to quiet substantially when all the unused input channels to the AVD card were referenced to a common ground. In efforts of ameliorating the noise problem some more, two possible $+5 \mathrm{~V}$ or greater inputs to the $\mathrm{A} / \mathrm{D}$ card were disconnected and the left over AD input channels were then referenced to the common ground. The $A / D$ card has a saturation level of $+-5 \mathrm{~V}$ and they are known to behave abnormally when they are saturated. Therefore we suspected that the existing noise spikes in all 12 of the output channels were caused by several of them saturating the A/D card, at which point the other channels start randomly tracking the saturated channels. After these possible saturation channels were removed from the AND card, the 6 remaining output channels stabilized at lower voltage magnitudes. To our surprise, after these improvements were made another significant noise source was identified. The laptop computer being used with the $A / D$ card was seen to affect the output channels as it was moved relative to the junction box. The box was closed with its plastic cover and moved as far as possible (as the A/D connector allowed) from the computer. Immediately after doing so, the readings stabilized down below $+1 \mathrm{~V}$. In fact, as the SixDOF system was turned on and off with all things unchanged, a consistent offset value was observed from the 6 channels of interest. This consistent voltage offset can be subtracted within the software so as to zero out the noise from all the channels when no light source is given to the PSDs. Following this great improvement of our signal processing system, it is expected to commence the calibration of the sensor very shortly. Once this step has been completed and the sensor is tested for linearity, a large-scale manufacturing operation of a working SixDOF sensor is the next item on the agenda. 


\section{Chapter 4}

\section{Conclusions and Recommendations}

In the previous section, large-scale manufacturing of the SixDOF sensor is mentioned as something that is being kept in mind. Many good reasons were given in sections 1 and 2 for the advent of the SixDOF sensor into the position-sensing market. A possible application of this SixDOF sensor that has yet to be mentioned has its roots in the large-scale design of the National Ignition Facility (NIF) taking place at Lawrence Livermore National Laboratory. NIF is currently a multi-billion dollar project to construct a mega-laser that will better serve our country's future energy requirements. In the design of NIF, an automated insertion vehicle is specified for use in the installation and possible cleaning of laser amplifier slabs and other critical optics. The NIF automation group working on the development of this automated vehicle is very interested in the SixDOF as an alternative position-sensing device for their existing sensors.

If the SixDOF sensor is to be implemented in the NIF automated Mobile Transport Vehicle (MTV) some new concerns arise with the design of the sensor that will require careful study. For instance, the current prototype of the sensor, by virtue of being crude, does not meet some of the accuracy requirements for the MTV. The NIF automated insertion process requires reliable detection of about $0.025 \mathrm{~mm}(0.001$ ") while the present status of the SixDOF only gives accuracy to about $0.5 \mathrm{~mm}$. Therefore, in order to meet these stringent requirements for the MTV the sensor has to be refined.

Perhaps, the most significant improvement that can be done to the SixDOF sensor is the replacement of the optics with more precisely manufactured optical mirrors, lenses, and beam splitters. This would have a drastic effect on the quality of the light spots on the PSDs and thus improve the detector's ability to discern more accurately the position data. An even greater concern has to do with the current filter used in the SixDOF sensor. When the sensor was built no exact specification was given as to its bandwidth curve. In other words, light of frequencies different from the laser light can be entering the sensor through its filter and degrading the accuracy of the PSD outputs. Since the PSDs have a spectral response range between $320 \mathrm{~nm}$ to $1100 \mathrm{~nm}$ it is very possible that these PSDs are detecting light other than the designed laser light. The filter in the SixDOF sensor is thought to be a notch filter enclosing the $670 \mathrm{~nm}$ spectral range. However, it is not known for sure. For NIF MTV applications, a very specific and reliable notch filter will be needed within the SixDOF sensor to provide very accurate position data. Based on the research done, these recommended changes are the most critical for the SixDOF sensor if it is to be used in the MTV design or in similar robotics applications. 


\section{Appendix A}

\section{Determination of the Calibration Matrix}

The decoupling of the SixDOF position-sensing device is really a means to calibrate the sensor so that when specific inputs are given ,the correct outputs are displayed automatically. The first operation in the calibration is to adjust the voltage of the laser to $3 \mathrm{~V}$. The power switches for the laser and for the signal conditioning boards are then turned on and the sensor readings are allowed to stabilize. Remember that the voltage offset is subtracted from the readings using the software. Using the six degrees of freedom positioner, the sensor head is moved a known distance in any direction for various directions and orientations. The position of the sensor head is then recorded and another sample is taken and its new position is recorded. The more samples one takes for the sensor calibration the more accurate the calibration matrix will be. This means improved linearity in the position data when it is verified. In the following derivation the number of samples will be assumed to be five.

To completely decouple the six degrees of freedom of information it is necessary to solve Equation 1 in section 4 . Equation 1 can be written in the following extended form:

$$
\left[\begin{array}{llllll}
c_{11} & c_{12} & c_{13} & c_{14} & c_{15} & c_{16} \\
c_{21} & c_{22} & c_{23} & c_{24} & c_{25} & c_{26} \\
c_{31} & c_{32} & c_{33} & c_{34} & c_{35} & c_{36} \\
c_{41} & c_{42} & c_{43} & c_{44} & c_{45} & c_{46} \\
c_{51} & c_{52} & c_{53} & c_{54} & c_{55} & c_{56} \\
c_{61} & c_{62} & c_{63} & c_{64} & c_{65} & c_{66}
\end{array}\right]\left[\begin{array}{lllll}
x_{1} & x_{2} & x_{3} & x_{4} & x_{5} \\
y_{1} & y_{2} & y_{3} & y_{4} & y_{5} \\
z_{1} & z_{2} & z_{3} & z_{4} & z_{5} \\
r_{x 1} & r_{x 2} & r_{x 3} & r_{x 4} & r_{x 5} \\
r_{y 1} & r_{y 2} & r_{y 3} & r_{y 4} & r_{y 5} \\
r_{21} & r_{22} & r_{z 3} & r_{24} & r_{z 5}
\end{array}\right]=\left[\begin{array}{lllll}
X_{1} & X_{2} & X_{3} & X_{4} & X_{5} \\
Y_{1} & Y_{2} & Y_{3} & Y_{4} & Y_{5} \\
Z_{1} & Z_{2} & Z_{3} & Z_{4} & Z_{5} \\
R_{x 1} & R_{x 2} & R_{x 3} & R_{x 4} & R_{x 5} \\
R_{y 1} & R_{y 2} & R_{y 3} & R_{y 4} & R_{y 5} \\
R_{z 1} & R_{22} & R_{z 3} & R_{24} & R_{z 5}
\end{array}\right]
$$

Where the matrix containing elements $c_{\mathrm{ij}}$ is the matrix $[\mathrm{C}]$, the matrix containing lower case deflection elements is $[\mathbf{S}]^{t}$, and the matrix containing the upper case deflection elements is $[D]^{t}$. To solve for the calibration matrix $[C]$ above relating the known deflections $X_{i}, Y_{i}$, $\mathrm{Z}_{\mathrm{i}}, \mathrm{R}_{\mathrm{xi}}, \mathbf{R}_{\mathrm{yi}}, \mathbf{R}_{\mathrm{zi}}$ to their corresponding sensor readings $\mathrm{x}_{\mathrm{i}}, \mathrm{y}_{\mathrm{i}}, \mathrm{z}_{\mathrm{i}}, \mathrm{r}_{\mathrm{xi}}, \mathrm{r}_{\mathrm{yi}}, \mathrm{r}_{\mathrm{zi}}$, we use Equation 2 in section 4.

Since the number of degrees of freedom, $k$, is the same as the effective number of sensors, $\mathrm{j}$, we can directly determine $[\mathrm{C}]$ from the inverse of the resulting $[\mathrm{CI}]^{t}$ matrix in Equation 2. Calibration is thus completed by observing the following, $[C I]^{-1}=[C]$. This procedure is similar to the one used by Cutkosky et al [3] to calibrate various force sensing devices located on a compliant wrist. 


\section{Appendix B}

\section{Wiring Schematics for Signal Processing Box}

The signal processing junction box encases all of the signal processing components of the SixDOF sensor system. Figure B1 describes in detail the connection between the PSDs and the signal processing circuit boards.

Figure B.1: Wiring schematic for PSDs and Boards

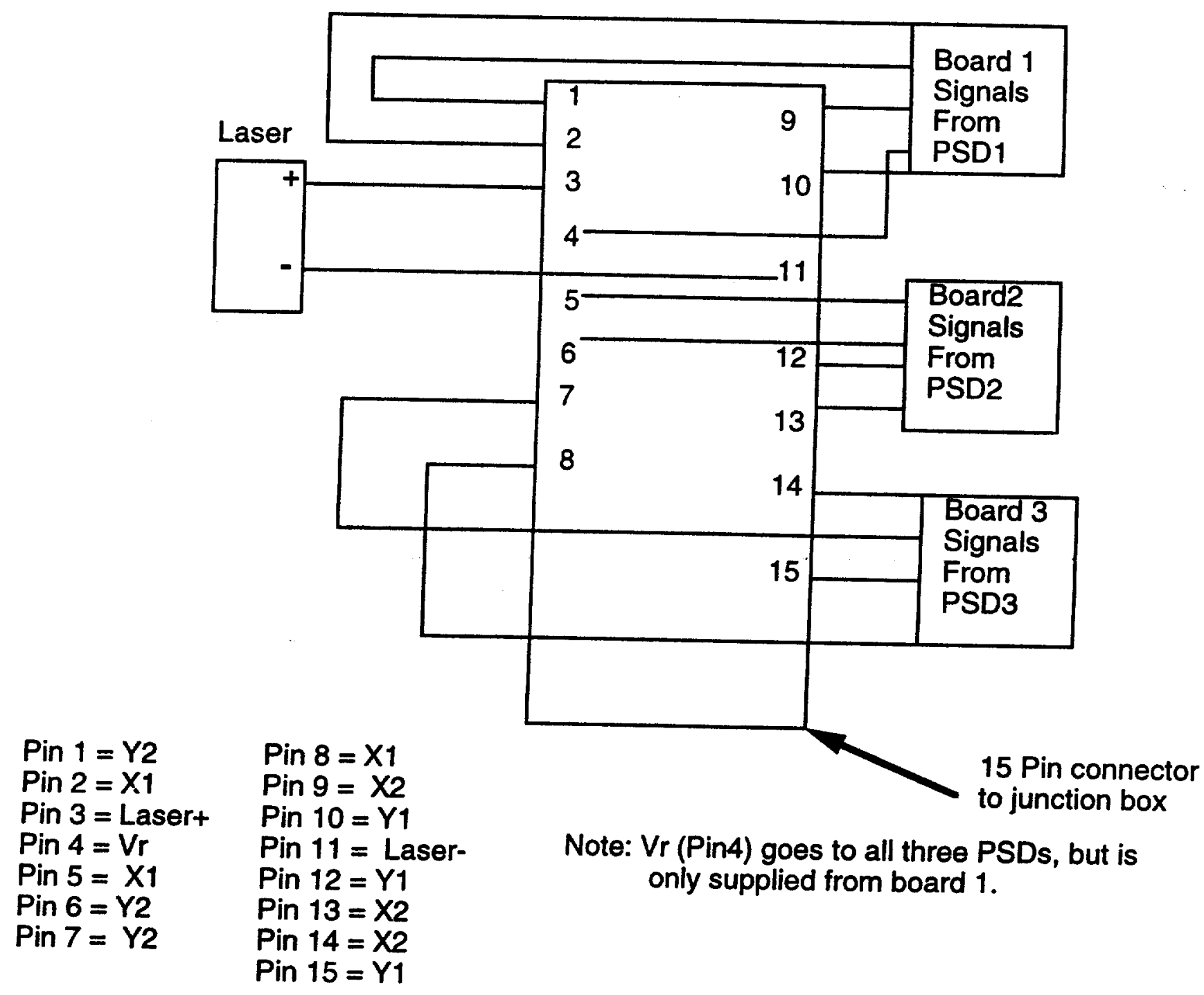


Figure B2 shows the wiring that was used for the board itself.

Figure B.2: Pin schematic for Signal Processing Boards

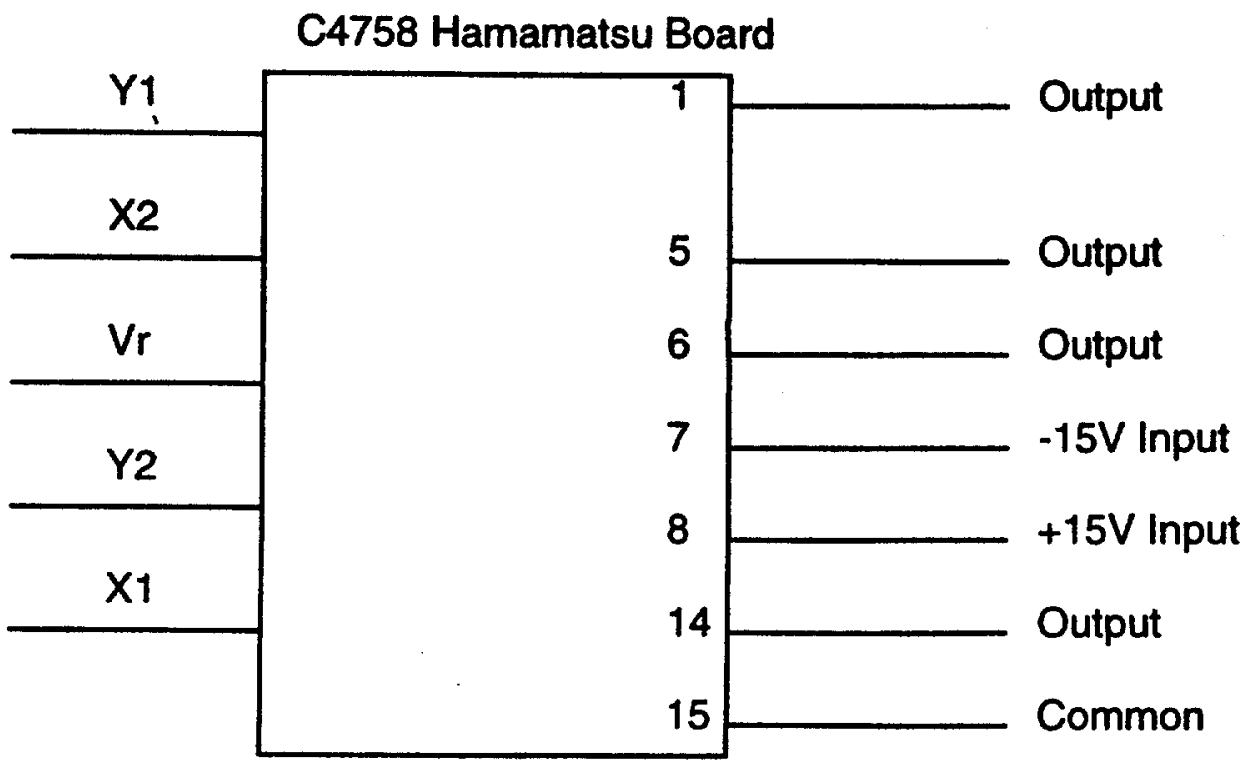

Figure B3 shows a schematic for the 37 pin connector used to link the junction box to the $A / D$ converter on the computer.

Figure B.3: Schematic of 37 pin connector

37 Pin Connector to AD

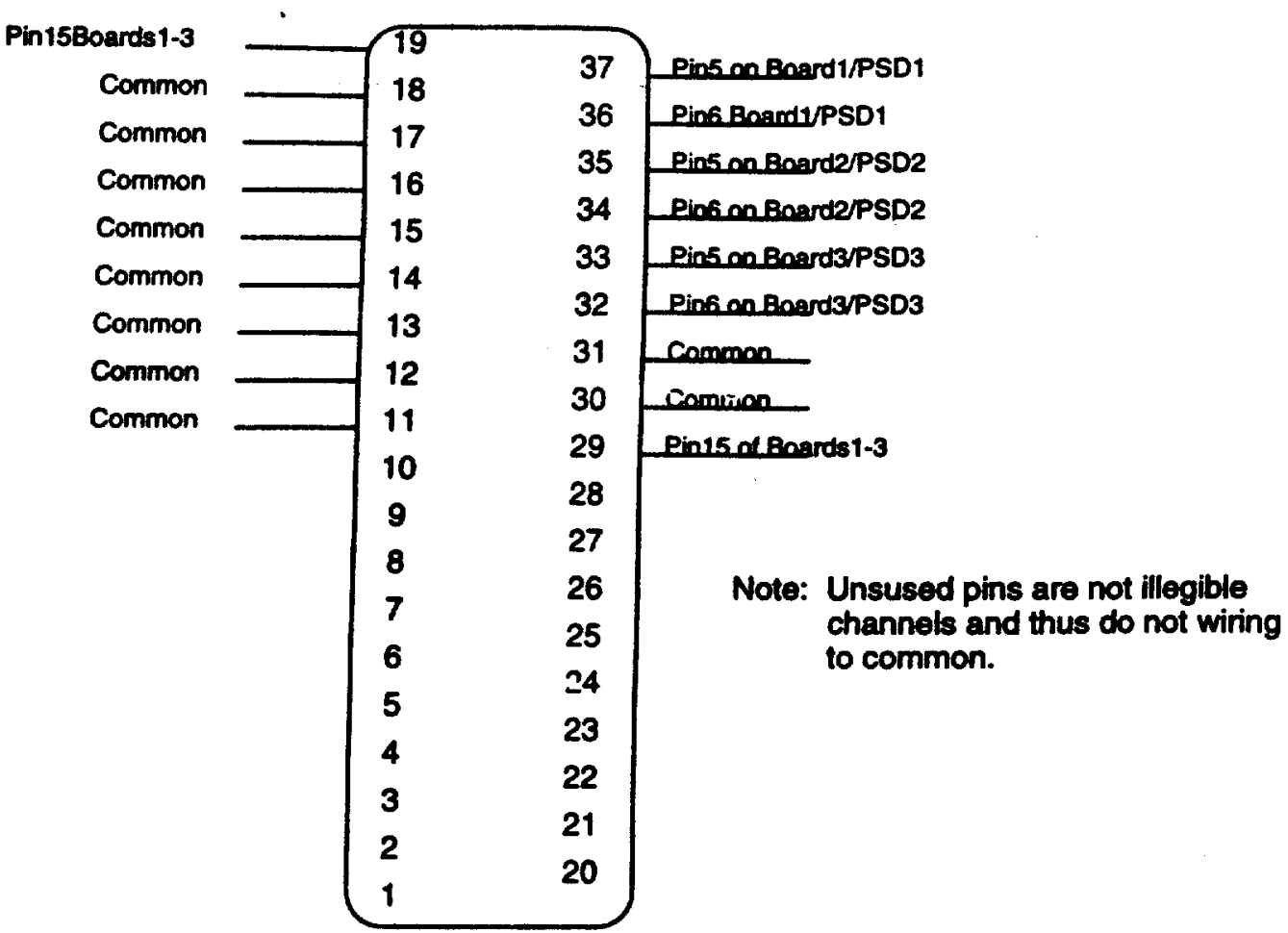




\section{References}

[1] C. Vann, SixDOF Sensor, 1996 R\&D 100 Award Entry Proposal.

[2] B.E. Shimano, The Kinematic Design and Force Control of Computer Controlled Manipulators, PhD thesis, Stanford University, March 1978.

[3] M.R. Cutkosky and P.K. Wright, "Position Sensing Wrists for Industrial Manipulators," Robotics Institute Technical Report CMU-RI-TR-82-9, Carnegie-Mellon University, July 1982. 


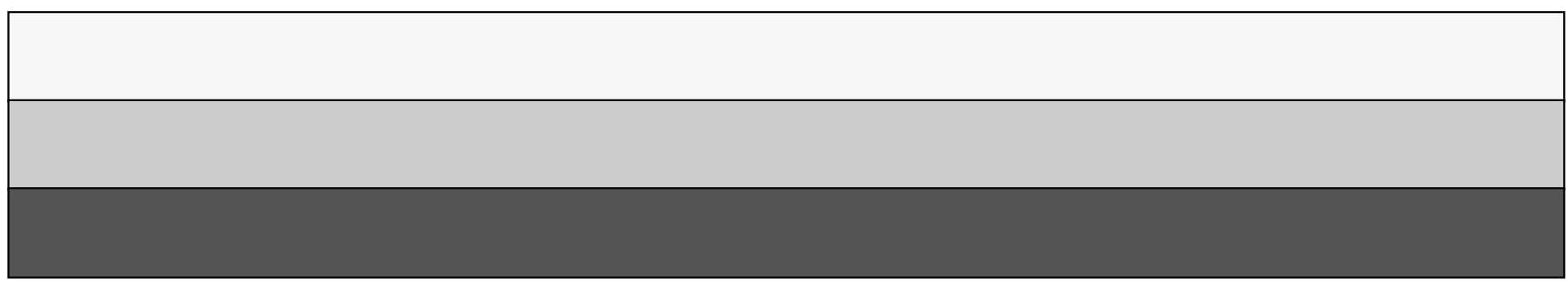

